EPJ Web of Conferences 103, 01010 (2015)

DOI: $10.1051 /$ epjconf/ 201510301010

(C) Owned by the authors, published by EDP Sciences, 2015

\title{
Spiral Light Beams and Angular Momentum of Radiation
}

\author{
A.V.Masalov \\ Lebedev Physical Institute, RAS
}

The problem of angular momentum density of electromagnetic radiation is discussed. In connection with the spiral of light beams, able to manipulate atoms, molecules and nanoparticles, the formulas for local values of the orbital and spin angular momenta of radiation are necessary at evaluating the effect of particle rotation.

The whole angular momentum of a light beam, which can be transferred from the radiation to absorbing body, is the integral of the "classical" expression $[\mathbf{r} \times[\mathbf{E} \times \mathbf{B}]]$ over the cross section of the beam. However, for the particles of sub-wavelength size the density of angular momentum is necessary in calculations. The expression $[\mathbf{r} \times[\mathbf{E} \times \mathbf{B}]]$ is not the density of the angular momentum of the radiation, as it does not contain spin angular momentum part, i.e. the part which is related to the state of light polarization. Calculation formulas for the density of angular momentum can be obtained by studying the effect of the field on the atomic oscillator. These formulas are consistent with the results of a rigorous quantum description of light [1].

Examples of spiral light beams with different polarization states of radiation are considered, in particular with radial and azimuthal polarization.

\section{Reference}

1. A.I. Akhiezer, V.B. Berestetsky. Quantum electrodynamics (John Wiley \& Sons, 1965)

This is an Open Access article distributed under the terms of the Creative Commons Attribution License 4.0, which permits unrestricted use, distribution, and reproduction in any medium, provided the original work is properly cited. 